\section{Fits, faints and funny turns}

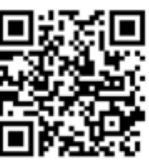

Fits, faints and funny turns represent a common reason for presentation - either to the general practitioner or to the emergency department. In many cases, the consultation is dissatisfying for the doctor and the patient, as such patients frequently present a diagnostic dilemma for the clinician. Frequently, the key to a satisfactory evaluation is a structured approach, premised on a clear and comprehensive history focused on prior comorbidities and the episode - its context, precipitating factors, prior situational factors, onset and evolution, and events occurring afterwards. A detailed and carefully elicited medical history allows the clinician to confirm the diagnosis, delineate the underlying mechanism, and identify features that may suggest high risk of recurrence, injury or death.

This issue of CME includes a series of articles on approaches to common causes of fits, faints and funny turns in adults (Table 1). The

Table 1. Common causes of fits, faints and funny turns in adults

\begin{tabular}{l}
\hline Epilepsy \\
Tonic, clonic, atonic seizures \\
Focal seizures with impaired awareness or secondary generalisation \\
Primary absence seizures \\
Generalised tonic-clonic seizures \\
Cardiac disease \\
Cardiomyopathy (HCM, ARVC) \\
Channelopathies (Brugada, CPVT, LQTS, SQTS) \\
Conduction abnormalities (2nd-, 3rd- and high-degree AV block) \\
Arrhythmias \\
Valvular heart disease (AS, MS) \\
Pericardial disease \\
Postural hypotension \\
Vasovagal/syncope \\
Carotid sinus hypersensitivity \\
Falls \\
Migraine variants \\
Vertigo \\
BPPV \\
Vestibular neuritis \\
Cerebellar stroke \\
Vestibular migraine \\
Ménière's disease \\
Drug reaction \\
Metabolic causes \\
Hypoglycaemia \\
Electrolyte abnormalities \\
Anaemia \\
Transient ischaemic attacks \\
Amnestic episodes \\
Cervical spondylosis \\
Sleep disorders \\
Sleep apnoea \\
Narcolepsy/cataplexy \\
Autonomic failure \\
\end{tabular}

Continued ..
Table 1. (continued) Common causes of fits, faints and funny turns in adults

\begin{tabular}{l}
\hline Psychogenic \\
Conversion reactions \\
Fugue states \\
Malingering \\
Personality disorders \\
Phobia/anxiety states \\
Psychoses/severe depression \\
Cultural/language conflicts \\
Hyperventilation \\
Drugs \\
Alcohol \\
Peripheral vasodilators (angiotensin-converting enzyme inhibitors, \\
angiotensin II receptor blockers, glyceryl nitrate, hydralazine, \\
prazosin) \\
Diuretics \\
Anti-epileptic drugs \\
Antihypertensive drugs \\
Barbiturates \\
Benzodiazepines \\
Phenothiazines \\
Phenoxybenzamine \\
Selective serotonin re-uptake inhibitors \\
Tricyclic antidepressants \\
OTC anticholinergic agents \\
ARVC= arrhythmogenic right ventricular cardiomyopathy; AS = aortic stenosis; AV = \\
atrioventricular; $;$ pPPV = benign paroxysmal positional vertigo: CPVT = catecholaminergic \\
paroxysmal ventricular tachycardia; HCM = hypertrophic cardiomyopathy; LQTS = long QT \\
syndrome; MS = mitral stenosis; OTC = over the counter; SQTS = short QT syndrome.
\end{tabular}

four articles emphasise the underlying mechanisms for such episodes and provide the busy clinician with a sound and simplified approach to the clinical evaluation and management.

In the first article, by Ntusi et al. ${ }^{[1]}$ the pathophysiology of and clinical approach to syncope are reviewed. The classification, which is based on the underlying pathophysiological mechanism causing the event, includes cardiac, orthostatic and reflex (neurally mediated) mechanisms. The prognosis of syncope relates to: $(i)$ the risk of death and life-threatening events; and (ii) the risk of recurrence of the syncope and physical injury. The history is key to the assessment of the patient with syncope. The routine use of a broad panel of laboratory tests is not recommended and tests should only be requested if clinically indicated; however, an ECG should be performed in all patients. The goals of management are to prolong survival, limit physical injuries and prevent recurrences. Patients with life-threatening causes of syncope should be managed urgently and appropriately.

In the second article, Lee-Pan and Tucker ${ }^{[2]}$ provide a comprehensive review of epilepsy in adults and introduce the reader to an updated classification of epilepsy. A Generalised seizure indicates a disorder in which the entire cortex instantly becomes epileptiform in nature, and may be classified as Absence or Generalised Tonic-Clonic Seizure (GTCS). Focal seizures may occur with retained or altered awareness, or evolve secondarily into a GTCS. While not always easy, it is fundamentally important to differentiate Generalised from Focal seizures, as the two have a different aetiology and pathophysiology, 
and the approach to management differs. A simplified schema for remembering different seizure types is provided, and a clinically useful $\mathrm{A}->\mathrm{B}->\mathrm{C}$ mnemonic is recommended for patients and medical staff.

In the third article, by Bateman et al., ${ }^{[3]}$ acute vertigo is discussed. Vertigo is usually benign and caused by peripheral vestibular disease. It is important to make a specific diagnosis, and the history should answer three questions: (i) Is it vertigo? (ii) Is the lesion central or peripheral? (iii) Does the patient require neuro-imaging? The different causes of acute vertigo and their management are considered in detail, including benign paroxysmal positional vertigo, vestibular neuritis, cerebellar stroke, vestibular migraine and Ménière's disease. Vestibular rehabilitation therapy may substantially improve the functioning of patients with vertigo, whereas long-term vestibular suppressants are likely to impede recovery.

In the last article, by De Villiers and Kalula, ${ }^{[4]}$ falls and balance problems in the elderly are revisited. Gait instability and falls are common in elderly persons and have devastating consequences, with substantial morbidity and mortality, and are a significant precipitant for functional decline. The causes of falls in elderly persons are usually multifactorial, with a combination of intrinsic factors, including physiological changes of ageing, frailty and pathologies, and extrinsic, environmental and situational factors. Maintaining postural control requires a complex integration of sensory input, central processing, motor co-ordination and musculoskeletal function, which decrease with ageing. The assessment and management of a patient who is at risk of falls or who has fallen require a multidisciplinary approach to identify and address factors contributing to the falls. The success of interventions to treat and manage these problems can be gauged by improvements in functional status. Prevention of falls focuses on maintaining mobility and balance and identifying those at risk of a fall for multidisciplinary assessment and intervention.

In conclusion, the patient presenting with a problem of a faint, fit or funny turn may present a diagnostic challenge for the busy general practitioner or junior (and often overwhelmed) emergency department doctor. A clear history is crucial for a thorough evaluation. It is my hope that this series of articles will improve not only the understanding of busy general practitioners, medical officers and emergency medicine doctors, but also decrease levels of anxiety among patients with such problems, who often feel frustrated that neither a cause nor a satisfactory explanation can be found for their problem to allay their fears.

\section{Ntobeko A B Ntusi}

Guest editor

ntobeko.ntusi@gmail.com

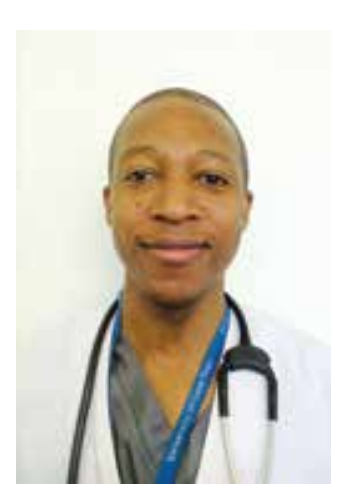

1. Ntusi NAB, Coccia CBI, Cupido BJ, Chin A. An approach to the clinical assessment and management of syncope in adults. S Afr Med J 2015;105(8):690-693. [http://dx.doi.org/10.7196/SAMJnew.8065] 2. Lee-Pan EB, Tucker L. An approach to epilepsy. S Afr Med J 2015;105(8):693. [http://dx.doi.org/10.7196/ SAMJnew.8039]

3. Bateman K, Rogers C, Meyer E. An approach to acute vertigo. S Afr Med J 2015;105(8):694. [http:// dx.doi.org/10.7196/SAMJnew.8097]

4. De Villiers L, Kalula SZ. An approach to balance problems and falls in elderly persons. S Afr Med J 2015;105(8):695. [http://dx.doi.org/10.7196/SAMJnew.8037]

S Afr Med J 2015;105(8):689. DOI:10.7196/SAMJnew.8098 